\title{
La situación del empleo en turismo rural en España
}

\author{
PEDRO ARIAS MARTÍN
}

Departamento de Economía y Ciencias Sociales Agrarias, UNIVERSIDAD POLITÉCNICA DE MADRID, ESPAÑA.E-mail: pedro.arias@upm.es

\section{RESUMEN}

En este trabajo se aborda el empleo en turismo rural, pues es una actividad cada vez más importante en dicho entorno $\mathrm{y}$, en muchos casos, complementa la renta de los agricultores, lo que también puede evitar la despoblación de estas áreas. Para realizar este estudio, se parte de un análisis del empleo bajo un enfoque general y agrario para finalmente definir el comportamiento mensual que ha seguido el empleo a nivel nacional, empleando técnicas de series temporales basadas en modelos ARIMA.

Palabras clave: Turismo rural, empleo, series temporales, modelos ARIMA.

\section{The Employment Situation in Rural Tourism in Spain}

\begin{abstract}
This article deals with the employment in rural tourism, as it is an increasingly important activity in these areas and it can prevent depopulation. For this, the tourism is treated and the employment is analyzed from the point of view general and agrarian, too. Finally, the monthly behavior of employment in rural tourism is defined at national level, using techniques based in ARIMA time series models.
\end{abstract}

Keywords: Rural Tourism, Employment, Time Series, ARIMA Models.

Clasificación JEL: C22, L83

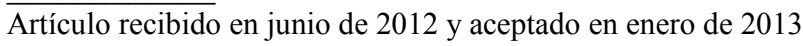

Artículo disponible en versión electrónica en la página www.revista-eea.net, ref. ə-31104 


\section{INTRODUCCIÓN}

El turismo es uno de los sectores más importantes en España. Según se recoge en el Plan Nacional e Integral de Turismo 2012-2015 del Ministerio de Industria, Energía y Turismo, España tiene el liderazgo mundial en turismo, es el primer destino del mundo en turismo vacacional, el segundo país por gasto turístico y el cuarto por el número de turistas. La actividad turística supone más de un $10 \%$ del PIB, crea un $11 \%$ del empleo y contrarresta, en gran medida, nuestro déficit comercial.

Esta situación hace que la Administración lo considere como un activo estratégico para la creación de riqueza y empleo, como reconocen también Yagüe (2002), Lillo-Bañuls y Casado-Díaz (2011), Juaneda y Riera (2011) o Cuñado et al (2011), que se comporte con gran dinamismo incluso en tiempos de crisis, que cuente con una gran capacidad de arrastre de otros sectores productivos y que sea un posible eje para la recuperación económica.

Si el turismo, desde un punto de vista global, es una actividad con cierta notoriedad en términos económicos, no menos lo es una parte de ese turismo dirigido al ámbito rural, por las connotaciones sociales que además provoca. En este sentido se manifiestan Cánoves et al (2000) señalando como ventajas sociales, el mitigar la emigración de las zonas rurales, el beneficiarse del intercambio cultural entre el mundo urbano y rural, el revalorizar los modelos de vida rural o el desarrollo más equilibrado de zonas más marginales, desfavorecidas y aisladas, que son en parte áreas poco conocidas.

Asimismo y bajo planteamientos similares el Ministerio de Medio Ambiente y Medio Rural y Marino (MARM, 2009), define el turismo rural como una actividad económica, con gran potencial en el medio rural, que favorece el asentamiento de la población, el crecimiento de su renta y su capacidad para contribuir a la conservación del patrimonio histórico, cultural y ambiental.

El turismo rural tiene cada vez más relevancia en estas zonas, como reconoce la OCDE (1994) en un documento sobre estrategias para el turismo y desarrollo rural. También, otros autores como Pulido y Cárdenas (2011) inciden en esa importancia, manifestando el crecimiento significativo que se ha producido en España en la última década. Estos autores justifican ese crecimiento tanto por factores externos (rechazo de la masificación ante las grandes concentraciones de turistas en el litoral, el creciente deseo de personalización de los viajes, el mayor interés general por el medio ambiente, así como la vuelta a los valores de la cultura local materializados en el mundo rural) como por factores internos, centrados en la decidida apuesta de la Administración por el turismo, como una oportunidad para la transformación de una estructura productiva del mundo rural obsoleta. Asimismo, Fernández-Cavada et al (2007, p.249) manifiestan esa relevancia del turismo señalando que: “... si como parece evidente la agricultura no puede ser en el futuro el sector económico capaz de mantener a la po- 
blación en el medio rural..., el Desarrollo Rural debe contemplar la potenciación de sectores económicos alternativos al agrario capaces de crear actividad económica con atractivo para lograr el mantenimiento y el crecimiento de la población rural, y en este sentido parece que a corto plazo el que más posibilidades tiene es el del turismo rural'.

En este contexto de creciente interés e importancia por estas actividades, el objetivo de este trabajo consiste en analizar el empleo generado por el turismo rural. Para ello, se estima el modelo de comportamiento mensual seguido por el personal empleado para el periodo 2001-2011. Se ha optado por este periodo de análisis por considerarlo más homogéneo, teniendo en cuenta que en España se produce el cambio de moneda, con la utilización del euro, y este hecho podría incidir en los datos sobre turismo.

El estudio se basa en informaciones proporcionadas por el Instituto Nacional de Estadística (INE) a través de la Encuesta de Población Activa (EPA) y de la Encuesta en Alojamientos de Turismo rural, así como en datos del Ministerio de Medio Ambiente y Medio Rural y Marino sobre turismo rural recogidos en Anuarios de Estadística Agraria.

Este trabajo pretende comparar la situación del empleo desde un punto de vista general, agrario así como en el turismo rural y analizar si su comportamiento está correlacionado en el tiempo. Además, se estima el modelo funcional de la serie temporal a través de los modelos ARIMA, metodología seguida por numerosos autores, como Song et al (2003), Song and Li (2008) o Mondejar (2007) que manifiestan que las series temporales constituyen una herramienta sencilla e ideal para analizar el turismo.

\section{EL TURISMO RURAL}

El turismo rural es una actividad que, si bien se ha desarrollado con gran intensidad en España en los últimos años, se ha producido desde hace tiempo. Inicialmente, este turismo, provocado por el movimiento de la población urbana hacia el campo, era minoritario y principalmente realizado por personas con un cierto arraigo rural (emigración, familiares...), aunque como señalaba Camilleri (1967) no se concebía como un factor de desarrollo rural, pues dicho desarrollo se centraba en la producción agraria.

Esta concepción del desarrollo rural ha evolucionado y también otras actividades se tienen en cuenta cuando se planifica el desarrollo rural de una zona. Es el caso del turismo rural, actividad que complementa la renta de los agricultores, como reconoce Carazo (1982, p.118) indicando: "Para la población agraria de estas áreas, todos esos elementos de atracción son recursos potenciales que, adecuadamente utilizados, pueden representar un incremento en sus ingresos económicos y convertirse en importante factor de desarrollo". 
Pero, además puede mantener a las personas en el entorno rural evitando su despoblación, como recogen Cercós (1973), OCDE (1994) o Cánoves et al (2005). Incluso, algunos autores como Brunet y Alarcón (2008, p.144) consideran al turismo rural como competidor de otras actividades agrarias, manifestando textualmente: “... inicialmente, esta actividad se concibió como un complemento de rentas a aquellas familias con actividad agrícola. Pero, en las últimas décadas, de ser una actividad complementaría a la agrícola ha pasado a ser un sector con entidad propia que llega, una vez se ha desarrollado el sector de turismo rural, a competir por la tierra y el trabajo en una misma área".

En esta evolución de la concepción del desarrollo rural, centrada en los años cincuenta en producir y abastecer de alimentos a la población, ha tenido un papel relevante la Administración, pues ha fomentado la incorporación de otras actividades, como el turismo. Por ello, en los años sesenta el gobierno español comienza a ser consciente de que los recursos turísticos eran una vía para mejorar los ingresos económicos e impulsar el desarrollo rural, por lo que procede a fomentar estas actividades en 1967, con el Programa Vacaciones en Casas de Labranza dependiente del Servicio de Extensión Agraria, que asesoraba a los agricultores sobre dichas actividades y de la Secretaría de Estado de Turismo, que proporcionaba ayudas y difusión para el fomento del turismo rural.

Durante dos décadas este Programa nacional trató de desarrollar el turismo rural orientado, sobre todo, al alojamiento en casas rurales. Sin embargo, a partir de los años 80, con la aprobación de los diferentes Estatutos de Autonomía, tiene lugar un cambio importante en España, pues se empieza a producir una descentralización en muchas áreas y en particular en el turismo. Por lo tanto, cada Comunidad Autónoma pasa a fomentar el turismo rural de forma diferente, potenciando en mayor o menor medida estas actividades.

Con la entrada de España en la UE el desarrollo rural y, en particular, el turismo rural se ha beneficiado de ayudas a través de diferentes fondos (FEOGA, FSE, FEDER...) y con las iniciativas comunitarias LEADER, complementadas en España con los programas PRODER. Todo ello, como señalan Calatrava y Sayadi (2004) ha provocado un fuerte impulso de dichas actividades en los últimos años.

En la actualidad el futuro del sector agrario está estrechamente ligado a la multifuncionalidad y al desarrollo equilibrado y sostenible de los territorios rurales. Las Administraciones Públicas han fomentado ejes de actuación para su consecución, recogidos en la política de desarrollo rural de la UE según el Reglamento (CE) 1698/05 relativo a la ayuda al desarrollo rural, a través del Fondo Europeo Agrícola de Desarrollo Rural (FEADER).

De esta forma el FEADER se ha convertido en el único fondo que financia directa y expresamente los programas de desarrollo rural, a través de una serie 
de medidas reunidas en torno a cuatro ejes. En concreto, en ellos se aborda el aumento de la competitividad del sector agrícola y forestal, la mejora del medio ambiente y del entorno rural, la calidad de vida en las zonas rurales y diversificación de la economía rural. Además, el Reglamento introduce un cuarto eje, de carácter transversal, donde se plantea la metodología LEADER.

Por consiguiente, bien a través de la metodología LEADER, bien a través del eje 3, orientado a objetivos específicos de diversificación de la economía rural (fomento de actividades turísticas,...) y de mejora de la calidad de vida en las zonas rurales se pretende que también el turismo sea una de las formas de potenciar las áreas rurales.

Además de estas ayudas comunitarias dirigidas, en general, al desarrollo rural y de las que se ha podido beneficiar, en particular, el turismo, España ha legislado medidas directamente dirigidas a potenciar el turismo rural. Es el caso del Plan Renove de turismo, iniciado en el año 2008, que persigue impulsar la rehabilitación de los establecimientos turísticos, mejorando la calidad y la sostenibilidad de las infraestructuras turísticas, con el objetivo de elevar el nivel y atractivo de la oferta turística.

También, otra línea de fomento del turismo rural es la Ley 45/2007 de 13 de diciembre, para el desarrollo sostenible del medio rural, que establece una nueva forma de enfocar el medio rural. En ella, se fijan las bases de la estrategia a seguir, bases cuyo contenido se amplía en el Programa de Desarrollo Rural Sostenible (2010-2014).

Es en este Programa de Desarrollo Rural Sostenible, cuyas acciones se articulan en torno a cinco ejes estratégicos, donde dentro del eje 1 "Actividad Económica y Empleo" se encuentra la medida de diversificación económica y, entre otras actividades, se recogen aquellas que generen riqueza y empleo en la zona, entre las que se encuentra el turismo rural.

En consecuencia, este apoyo institucional al turismo rural ha originado un fuerte impulso de estas actividades, pues el número de alojamientos, plazas, turistas y pernoctaciones ha aumentado constantemente, como se desprende de la comparación de la situación en el periodo 2011 respecto al 2001, donde se observa que, en general, se ha duplicado el número de turistas y de pernoctaciones, mientras que, el número de alojamientos y plazas han experimentado un crecimiento superior (Tabla 1).

Pero esta potenciación general del turismo ha tenido y tiene un efecto diferente dependiendo de la zona geográfica. La transferencia de competencias es una de las causas de esta heterogeneidad, como así lo reconoce el Ministerio de Industria, Energía y Turismo en su Plan Nacional e Integral de Turismo 20122015, incidiendo en la problemática desatada por la pluralidad de entes reguladores y la enorme dispersión en la regulación del sector. 
Tabla 1

Turismo rural, evolución a nivel nacional (número, periodo 2001-2011)

\begin{tabular}{|l|c|r|r|c|}
\hline Año & Alojamientos & \multicolumn{1}{c|}{ Plazas } & \multicolumn{1}{c|}{ Turistas } & Pernoctaciones \\
\hline 2001 & 5.497 & 42.925 & 1.210 .891 & 3.660 .815 \\
\hline 2002 & 6.004 & 51.010 & 1.370 .369 & 4.104 .680 \\
\hline 2003 & 6.974 & 59.884 & 1.467 .539 & 4.476 .140 \\
\hline 2004 & 8.234 & 71.488 & 1.754 .360 & 5.492 .516 \\
\hline 2005 & 9.629 & 83.916 & 1.982 .902 & 6.306 .329 \\
\hline 2006 & 10.830 & 95.906 & 2.425 .429 & 7.438 .393 \\
\hline 2007 & 11.559 & 103.455 & 2.661 .357 & 7.969 .361 \\
\hline 2008 & 12.794 & 114.766 & 2.623 .351 & 7.843 .924 \\
\hline 2009 & 13.886 & 126.222 & 2.714 .265 & 7.920 .437 \\
\hline 2010 & 14.320 & 130.882 & 2.647 .370 & 7.615 .988 \\
\hline 2011 & 15.035 & 137.761 & 2.715 .984 & 7.696 .366 \\
\hline
\end{tabular}

Fuente: INE (Encuesta de Ocupación en Alojamientos de Turismo Rural).

Paralelamente, esta transferencia ha dado lugar a que cada Administración fomente, en mayor o menor medida, el turismo y en particular el turismo rural. Pero, a pesar de ello, otro aspecto a tener en cuenta en el diferente desarrollo del turismo rural, es la extensión de la propia Comunidad Autónoma y la existencia de parajes con cierto encanto ambiental, pues son factores que repercuten en el desarrollo de esta actividad en ciertas Autonomías. Así, si se consideran ciertas variables, como alojamientos, plazas, turistas, pernoctaciones o empleo, para identificar el desarrollo del turismo rural en determinadas zonas se observa que en algunas Autonomías, entre ellas Castilla y León, Cataluña, Castilla-La Mancha, Asturias, Andalucía, Aragón o la C. Valenciana, esta actividad es más relevante que en las restantes (Tabla 2).

Tabla 2

Turismo rural, nivel autonómico (número, periodo 2011)

\begin{tabular}{|l|r|r|r|r|r|}
\hline \multicolumn{1}{|c|}{ Autonomías } & Alojamientos & Plazas & Turistas & Pernoctaciones & $\begin{array}{r}\text { Personal } \\
\text { ocupado }\end{array}$ \\
\hline Andalucía & 1.342 & 10.920 & 147.929 & 511.620 & 2.108 \\
\hline Aragón & 1.111 & 8.552 & 126.698 & 404.090 & 1.242 \\
\hline Asturias & 1.345 & 11.882 & 180.490 & 668.544 & 1.625 \\
\hline Baleares & 203 & 3.554 & 123.693 & 593.946 & 823 \\
\hline Canarias & 681 & 4.033 & 57.151 & 349.234 & 983 \\
\hline Cantabria & 395 & 6.836 & 192.612 & 475.951 & 615 \\
\hline Castilla y León & 3.188 & 28.807 & 625.371 & 1.421 .687 & 4.746 \\
\hline Castilla-La Mancha & 1.381 & 11.225 & 173.077 & 401.826 & 2.175 \\
\hline Cataluña & 1.732 & 13.848 & 311.942 & 927.964 & 2.239 \\
\hline
\end{tabular}


Tabla 2 (continuación)

Turismo rural, nivel autonómico (número, periodo 2011)

\begin{tabular}{|l|r|r|r|r|r|}
\hline \multicolumn{1}{|c|}{ Autonomías } & Alojamientos & Plazas & Turistas & Pernoctaciones & $\begin{array}{r}\text { Personal } \\
\text { ocupado }\end{array}$ \\
\hline C. Valenciana & 991 & 9.250 & 119.356 & 344.147 & 1.462 \\
\hline Extremadura & 473 & 5.561 & 113.556 & 263.318 & 718 \\
\hline Galicia & 535 & 6.591 & 143.235 & 295.003 & 894 \\
\hline Madrid & 223 & 3.880 & 118.364 & 224.008 & 508 \\
\hline Murcia & 272 & 2.984 & 24.234 & 73.651 & 360 \\
\hline Navarra & 677 & 4.869 & 115.693 & 363.328 & 876 \\
\hline País Vasco & 382 & 3.983 & 116.553 & 311.192 & 493 \\
\hline La Rioja & 106 & 987 & 26.030 & 66.857 & 123 \\
\hline
\end{tabular}

Fuente: INE (Encuesta de Ocupación en Alojamientos de Turismo Rural).

\section{ANÁLISIS DEL EMPLEO}

El fomento a la creación de empleo se ha convertido en un factor prioritario para nuestras instituciones en los últimos años. Tanto a nivel comunitario como nacional se han elaborado normativas donde se pone especial énfasis en su creación. Una de esas disposiciones en el ámbito rural es el Reglamento (CE) 1698/2005 que traslada a los Estados miembros la realización de Planes Estratégicos Nacionales. En el caso de España, este plan ha permitido, en primer lugar, diagnosticar los problemas a los que se enfrenta el medio rural, entre ellos, la despoblación y el envejecimiento y seguidamente, plantear las actuaciones para hacer frente a dichos problemas, entre las que se encuentra la creación de empleo.

En este contexto de apoyo, el análisis de la situación del empleo en el entorno agrario $\mathrm{y}$, en particular, el dedicado a turismo rural toma cierta relevancia de cara a estimar y predecir su comportamiento futuro.

Pero, para diagnosticar el empleo generado por el sector turístico en el entorno rural, es conveniente conocer la situación laboral de España a nivel general y en la que se encuentra el sector agrario, pues servirán de referencia para poder comparar, además, tendencias y comportamientos.

\subsection{La situación laboral. Población activa y ocupada}

El punto de partida para analizar la situación laboral en España es la Encuesta de Población Activa (EPA), elaborada por el Instituto Nacional de Estadística (INE). La información estadística recogida en ella es de gran interés, pues permite conocer diversas variables de carácter laboral. Entre estas se encuentran la población activa y la ocupada, con un comportamiento dispar en los últimos años y concretamente a partir del 2008. Este comportamiento ha estado 
marcado por la crisis económica donde la población activa y la ocupada han tomado tendencias diferentes. Por un lado la población activa se ha estabilizado, mientras que la ocupada ha sufrido una fuerte caída. Esta circunstancia, ha llevado a una situación preocupante a nivel general, pues a finales del 2011 la población activa se situaba en torno a 23 millones de personas, pero con una tasa de paro que ya superaba el 23\% (Figura 1).

Figura 1

Población activa y ocupada

(miles de personas)

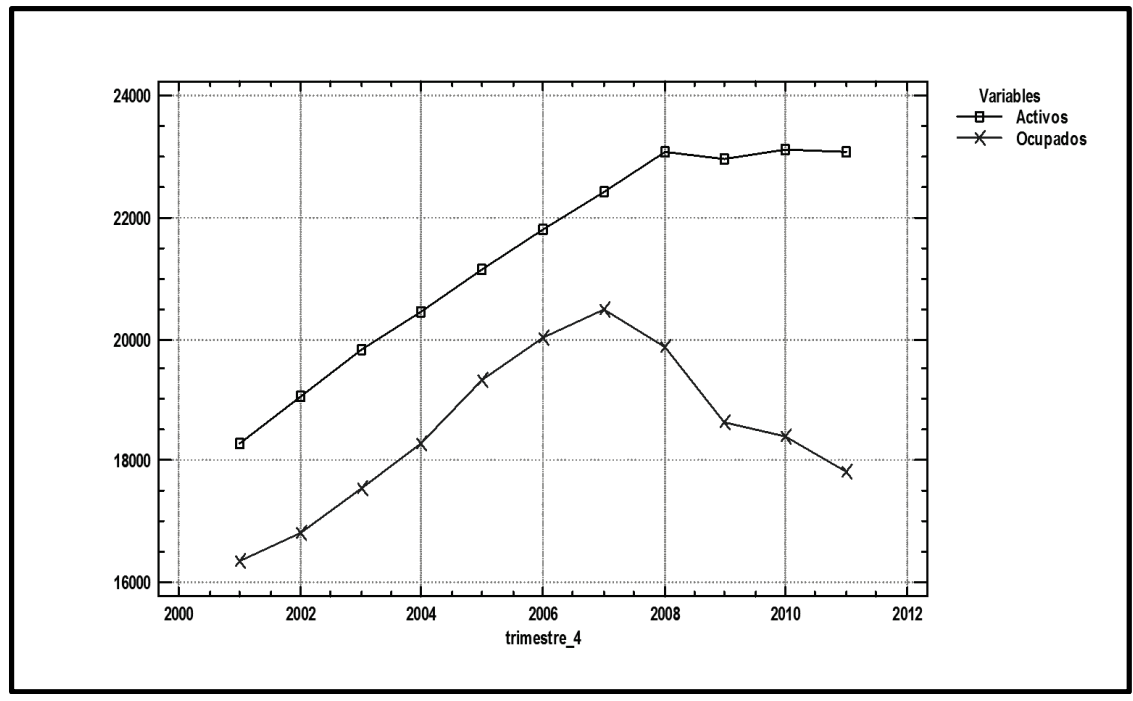

Fuente: Elaboración propia.

Con esta situación, y con unas previsiones nada favorables, el gobierno español ha tomado medidas, entre ellas, la reforma laboral del año 2012, recogida en el Real Decreto-Ley 3/2012 de 10 de febrero, que pretende fomentar la creación de empleo y, por lo tanto, reducir la tasa de paro que, en estos momentos, supone unas cifras un tanto alarmantes y muy lejanas de las que se presentan otros paises de la UE, cuya media comunitaria se sitúa en torno al $10 \%$.

Esta situación general es compartida por el sector agrario, que está inmerso en esta misma tendencia de pérdida de empleo, pues su población activa y el personal ocupado se han reducido a lo largo de la última década. Escudero (1986) justificaba esta reducción de población activa agraria al abandono de la actividad por parte de los agricultores, ya fuese por jubilación o por trasvase a otros sectores, mientras que otros, como Garrido y Gonzalez (1992) señalaban 
como motivos la política histórica de reducción del número de explotaciones y la intensificación de la mecanización en detrimento de personal contratado (Figura 2).

Figura 2

Población activa y ocupada en el sector agrario (miles personas, 2001-2011)

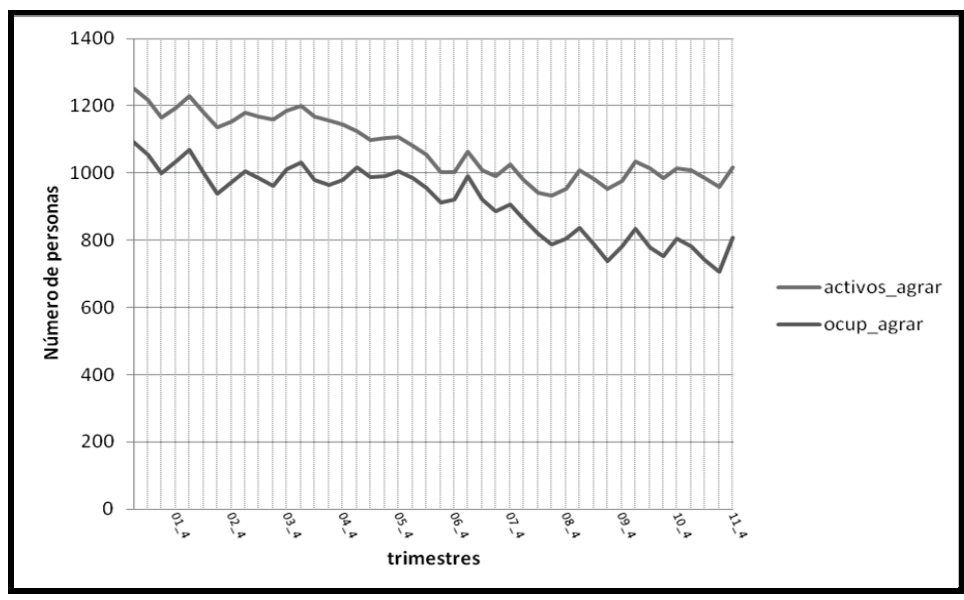

Fuente: Elaboración propia.

Pero, esta situación en el sector agrario no es coyuntural sino estructural, pues se viene padeciendo desde los años 60 , con una disminución sistemática de la población activa agraria y una importancia cada vez menor en el total de la población activa. Lo mismo ocurre con la población ocupada agraria que, cada vez, es más reducida y representa una pequeña parte de la total ocupada (en el periodo 2001-2011, entre el 3,8 y el 6,8\%).

$\mathrm{Y}$ es que el sector agrario se ha visto inmerso en diversas reformas de su política agraria, como es el caso de la percepción de una ayuda única por explotación, independiente del nivel de producción o como la vinculación de las ayudas al cumplimiento de ciertas normas medioambientales. Estas reformas, junto con la reducción de presupuestos comunitarios, han llevado a las explotaciones a una pérdida de renta $y$, en algunos casos, al abandono de dichas explotaciones, lo que ha repercutido en la pérdida de empleo.

Todas estas circunstancias hacen pensar en la existencia de un futuro un tanto incierto en el sector agrario, donde la competencia es cada vez más globalizada. Por ello, la actividad turística puede ser una alternativa a esta situación, ya no solamente en términos de renta sino también en el mantenimiento y en la creación de empleo. 


\subsection{Análisis del personal empleado en turismo rural}

El análisis del personal empleado se ha realizado aplicando la metodología de series temporales. Esta técnica data de los años setenta y fue desarrollada por Box y Jenkins y desde entonces, ha sido aplicada extensamente y en muy distintos campos. Es definida, entre otros, por Vandaele (1983) o Hoff (1983) como un conjunto de observaciones generadas secuencialmente a través del tiempo, con la característica de que los datos están ordenados respecto al tiempo y la existencia de una cierta dependencia o inercia entre las observaciones.

Uno de esos campos en el que se ha aplicado es el turismo, cuya creciente relevancia ha originado la proliferación de artículos que, como técnica de predicción, aplican series temporales, entre ellos, Bru y Usach (1987), Morley (1993), Hultkrantz and Olsson (1997), Lim and McAleer (2001), Goh et al (2002), Gil-Alana et al (2008), Song and Li (2008), Gil-Alana (2010), Cuñado et al (2011), Chang-Jui Lin et al (2011), Kader y Chikr-el-Mezouar (2011), Nanthakumar (2012) o Bigovic (2012). Una parte importante de ellos se centran en la demanda turística, como indica Wanhill (2011), aunque también se tratan otras variables, como pernoctaciones, llegadas de turistas, la industria del turismo,...

$\mathrm{Y}$ es que esa relevancia se debe a que la actividad turística constituye un factor con un gran potencial, no solo económico o de renta sino también por el empleo que genera MARM (2009). En este sentido se constata ese potencial con el crecimiento del número de personas empleadas, cuya cifra se ha multiplicado por tres en la última década (Figura 3).

Figura 3

Evolución del personal ocupado en turismo rural (media anual)

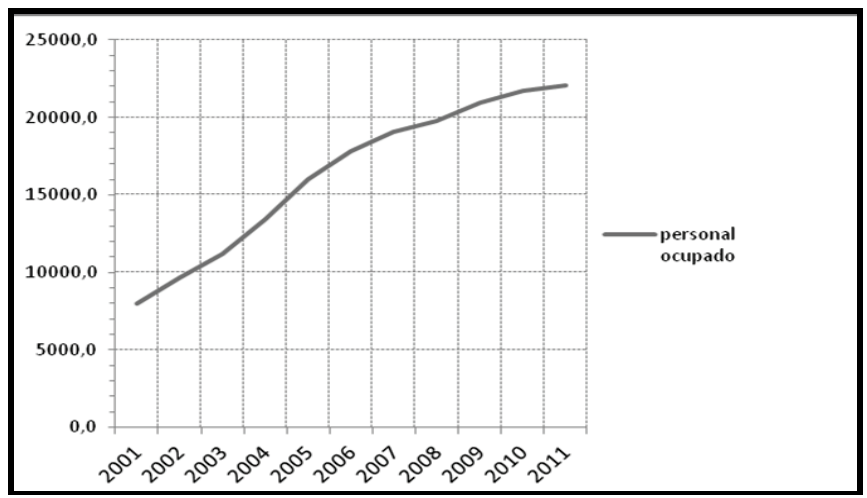

Fuente: Elaboración propia. 
Sin embargo, este crecimiento del personal empleado en turismo rural, que parece continuo, presenta variaciones cuando se analiza a nivel mensual. Un análisis descriptivo pone de manifiesto la existencia sistemática de un comportamiento estacional, con máximos en los meses de julio y agosto y mínimos a principios de cada año. Pero, además, presenta otras componentes, como tendencias y movimientos irregulares que se tratarán de identificar para estimar la serie temporal mediante los modelos ARIMA.

En cuanto a la tendencia de la serie se ha de indicar que, una estimación a través de una media móvil de orden 12 centrada, refleja un comportamiento creciente en el número de personas ocupadas en turismo rural.

Por su parte, el análisis de la estructura estacional se ha realizado considerando su comportamiento mensual para cada año y el que tiene cada mes para diferentes años. Este análisis mensual verifica un crecimiento medio en el número de personas empleadas desde enero hasta los meses de verano (julioagosto), periodo a partir del cual existe un cambio de tendencia, comportamiento que se reproduce para los diferentes años (Figura 4).

Figura 4

Componente de ciclo-tendencia para personal ocupado

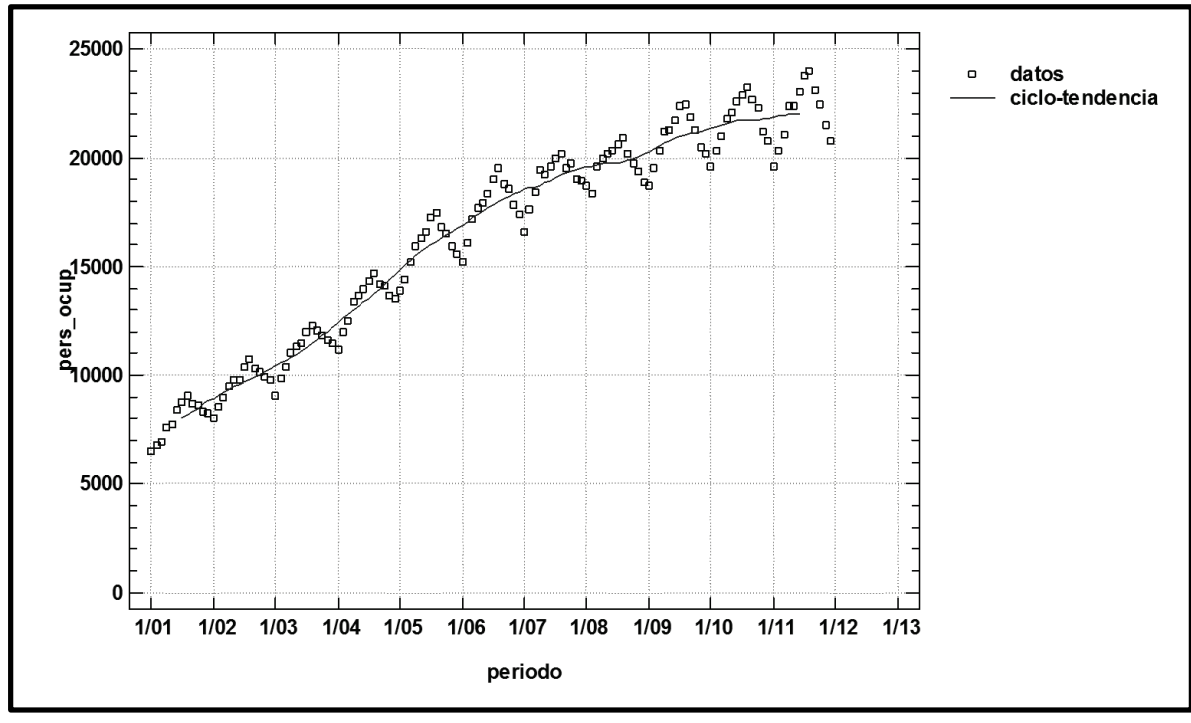

Fuente: Elaboración propia.

Del mismo modo, la estructura de cada mes para los diferentes años manifiesta un crecimiento del personal empleado en cada mes a medida que han transcurrido los años (Figura 5). 
Figura 5

Subseries estacionales para personal ocupado

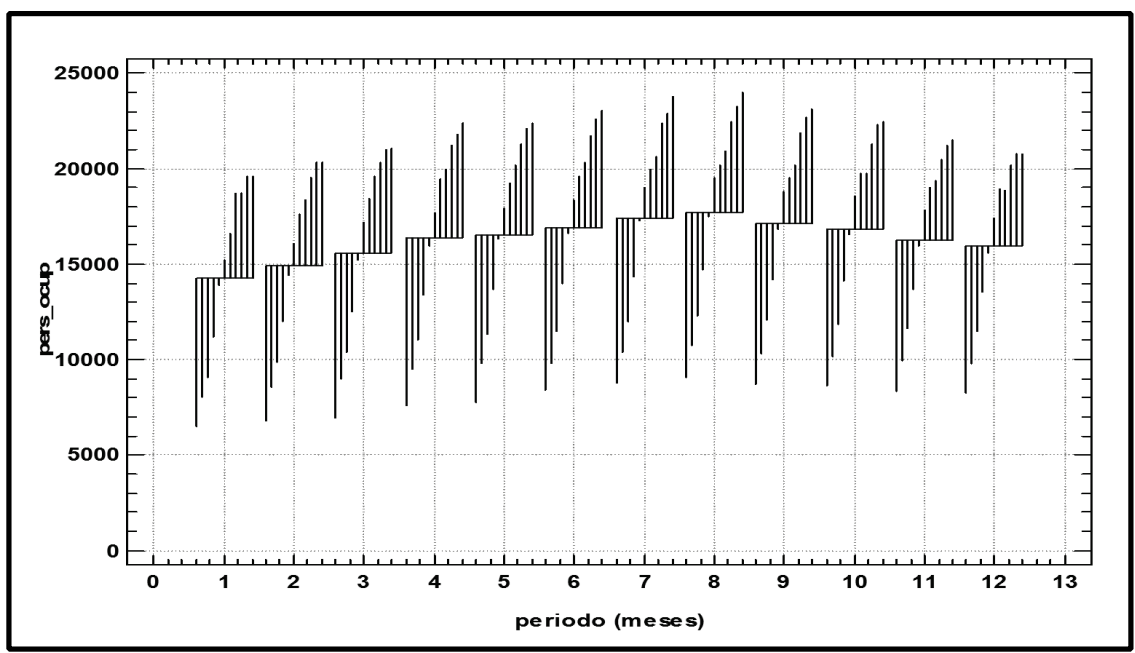

Fuente: Elaboración propia.

En consecuencia, este comportamiento induce a pensar en una falta de estacionariedad en la serie considerada. Por ello, es necesario analizar dicha característica y realizar las transformaciones oportunas como paso previo a la identificación y estimación del modelo.

Figura 6

Relación de la media respecto a la desviación típica

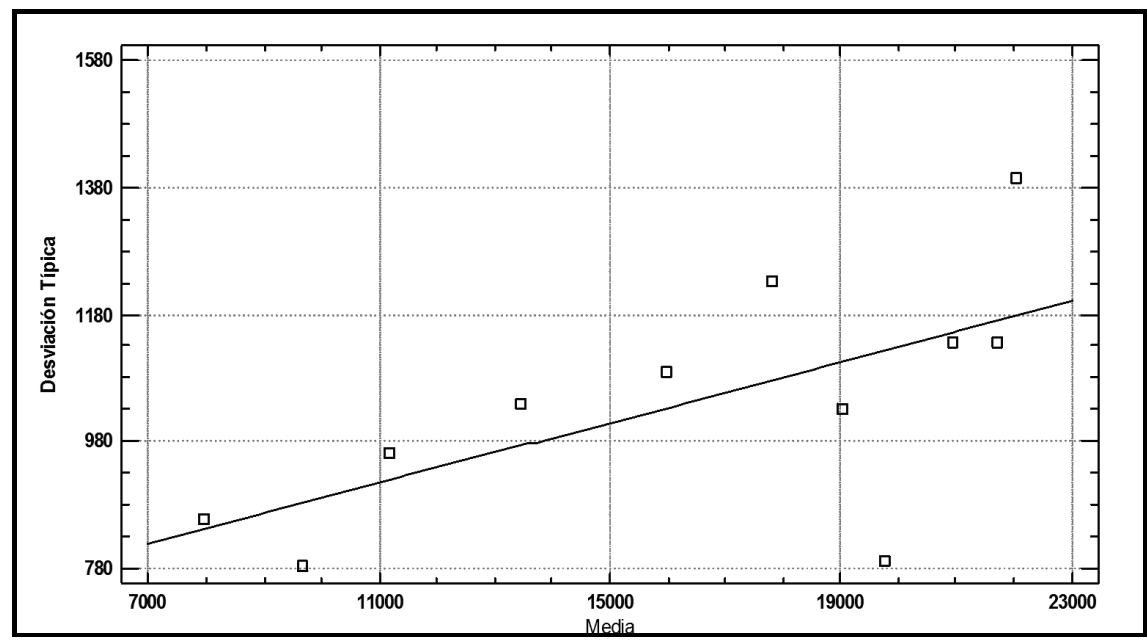

Fuente: Elaboración propia. 
La conclusión a la que se llega es que la serie original de empleados no es estacionaria ni en media ni en varianza, como se deduce de la representación de la media frente a la desviación típica, pues al crecer la media aumenta su desviación típica (Figura 6).

Igualmente, otro indicador que refleja esa falta de estacionariedad se obtiene al representar la función de autocorrelación, pues al aumentar el retardo los coeficientes no descienden rápidamente (Figura 7).

Figura 7

Autocorrelaciones estimadas para personal ocupado

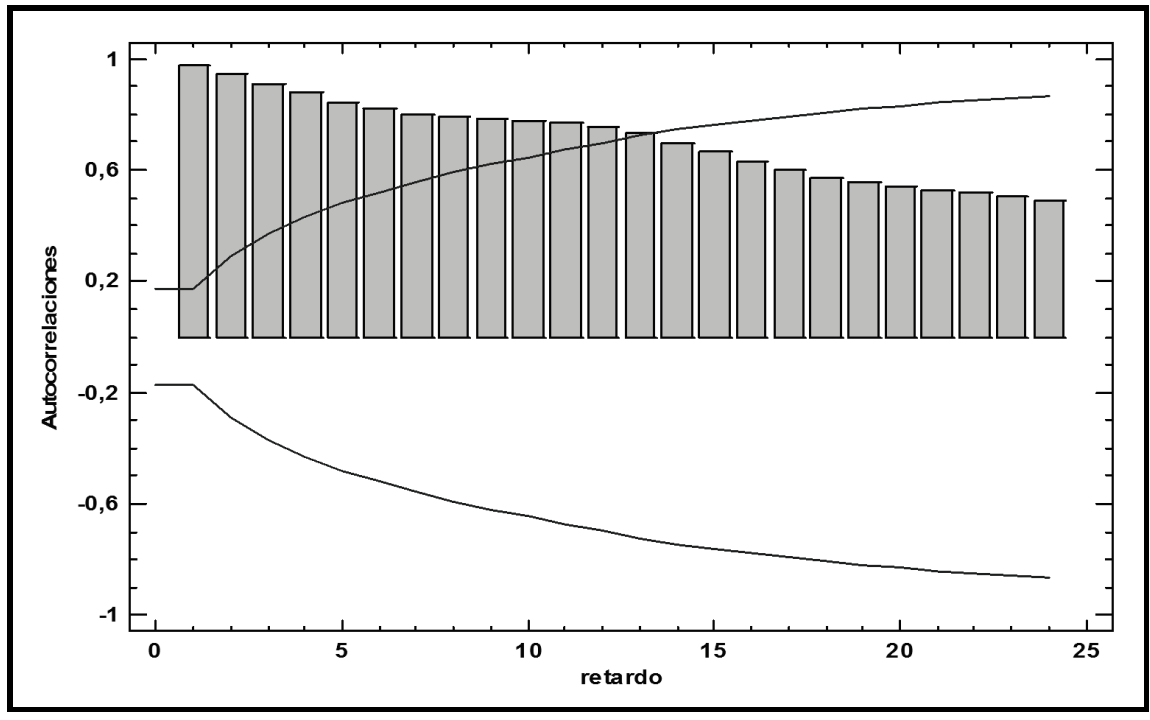

Fuente: Elaboración propia.

Por lo tanto, para conseguir la estacionariedad es necesario modificar la serie original, a través de una transformación logarítmica (para conseguir su homocedasticidad) y de una diferenciación regular y estacional. Estas transformaciones hacen que la serie, así modificada, tenga un comportamiento normal ${ }^{1}$ (Figura 8).

A partir de esta serie transformada se representan las funciones de autocorrelación simple y parcial. Estas funciones manifiestan la posibilidad de que el modelo presente una parte regular y de otra estacional, constituidas por un AR y un SMA de orden 1 respectivamente (Figuras 9 y 10).

\footnotetext{
${ }^{1}$ El contrate de Kolmogorov-Smirnov no ha rechazado la hipótesis nula (p-value: 0,245).
} 
Figura 8

Personal ocupado (turismo rural)

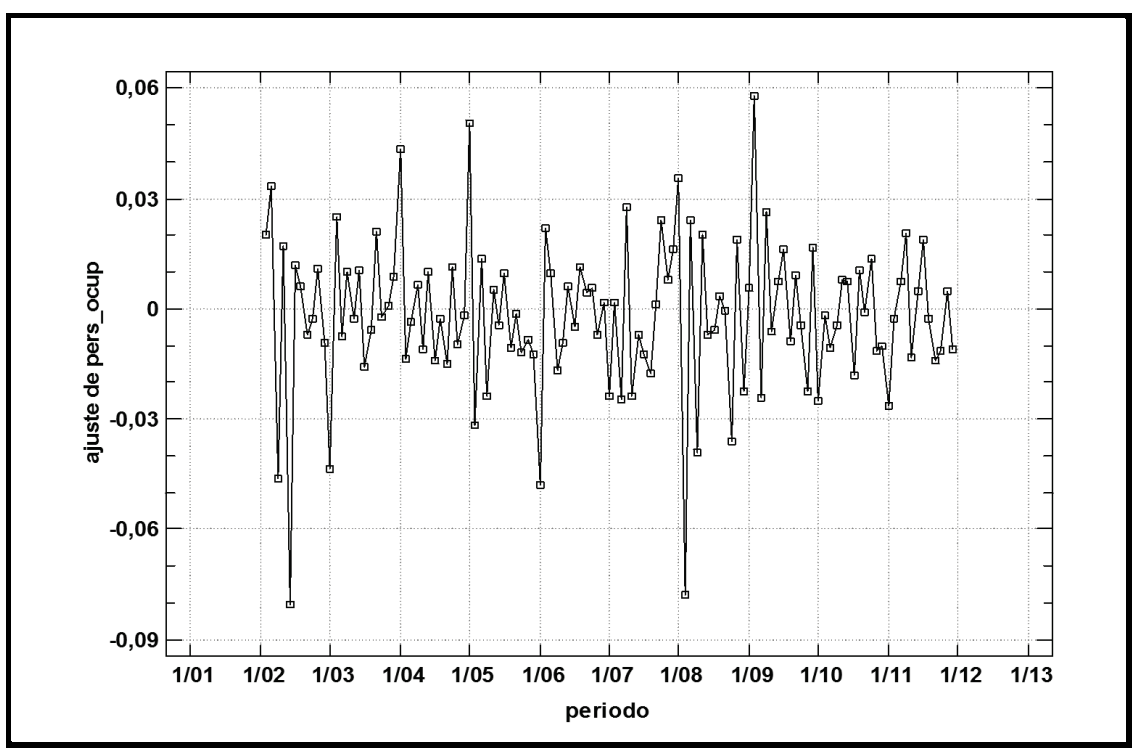

Fuente: Elaboración propia.

Figura 9

Autocorrelaciones estimadas para personal ocupado

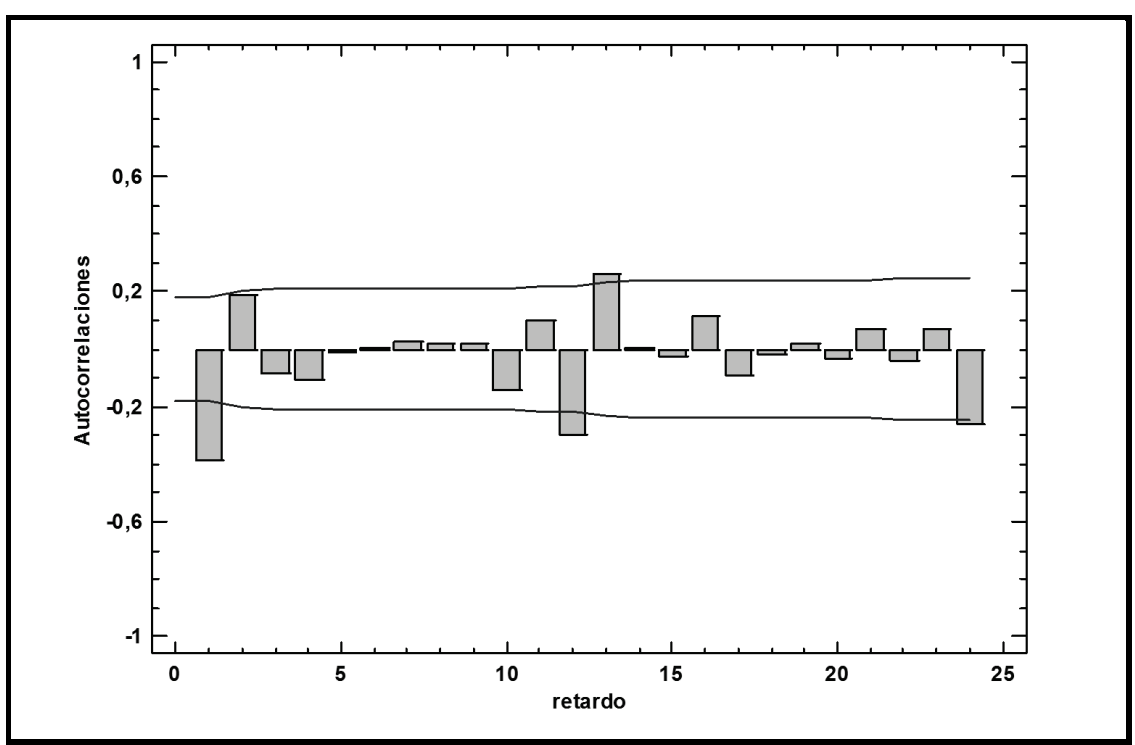

Fuente: Elaboración propia. 
Figura 10

Autocorrelaciones parciales estimadas para personal ocupado

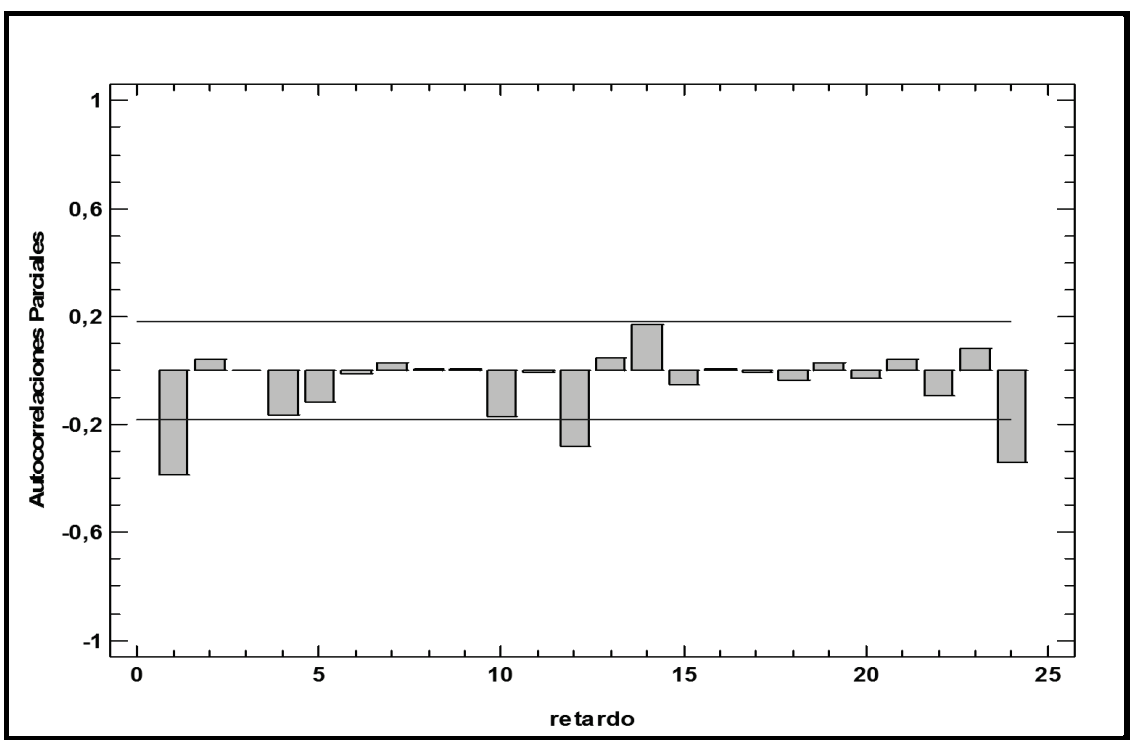

Fuente: Elaboración propia.

Seguidamente, y tras la identificación del modelo estimado se contrasta la validez de las diferentes hipótesis en las que se soporta dicho modelo. El resultado de este contraste verifica la elaboración de un modelo estacionario e invertible, el comportamiento de los residuos como ruido blanco (según contraste individual y global de Box-Pierce), la existencia de estabilidad en varianza y la significatividad de los coeficientes estimados.

Por lo tanto, el modelo estimado es un ARIMA $(1,1,0) \times(0,1,1)_{12}$ con constante, cuyos coeficientes y significatividad se recogen en la tabla 3.

Tabla 3

Estimación del Modelo ARIMA

\begin{tabular}{|l|c|c|c|r|}
\hline \multicolumn{1}{|c|}{ Parámetro } & Estimado & Error Estándar & $\mathbf{t}$ & Valor-P \\
\hline $\mathrm{AR}(1)$ & $-0,292509$ & 0,0890157 & $-3,28603$ & 0,001344 \\
\hline $\mathrm{SMA}(1)$ & 0,893347 & 0,0340913 & 26,2046 & 0 \\
\hline Media & $-0,001771$ & 0,0003254 & $-5,44434$ & 0 \\
\hline Constante & $-0,002290$ & & & \\
\hline
\end{tabular}

Fuente: Elaboración propia.

Y los errores en los que se ha incurrido en su obtención son (Tabla 4): 
Tabla 4

Errores del modelo estimado

\begin{tabular}{|c|c|c|c|c|c|}
\hline Estadístico & $\begin{array}{c}\text { Cuadrático } \\
\text { Medio }\end{array}$ & $\begin{array}{c}\text { Absoluto } \\
\text { Medio }\end{array}$ & $\begin{array}{c}\text { Absoluto } \\
\text { Porcentual } \\
\text { Medio }\end{array}$ & Medio & $\begin{array}{c}\text { Medio } \\
\text { Porcentual }\end{array}$ \\
\hline Estimación & 243,073 & 171,489 & 1,022 & $-2,979$ & $-0,019$ \\
\hline
\end{tabular}

Fuente: Elaboración propia.

\section{PREDICCIÓN DEL EMPLEO SEGÚN EL MODELO ESTIMADO}

La predicción es una etapa importante, pues toda la información que se ha utilizado para estimar el modelo se empleará, también, en prever la tendencia futura de la variable analizada. Así, según el modelo considerado y respecto al año 2011, existe una pérdida del número de personas ocupadas en turismo rural, como se recoge en la Figura 11.

Figura 11

Predicción para pers_ocup ARIMA $(1,1,0) \times(0,1,1) 12$ con constante

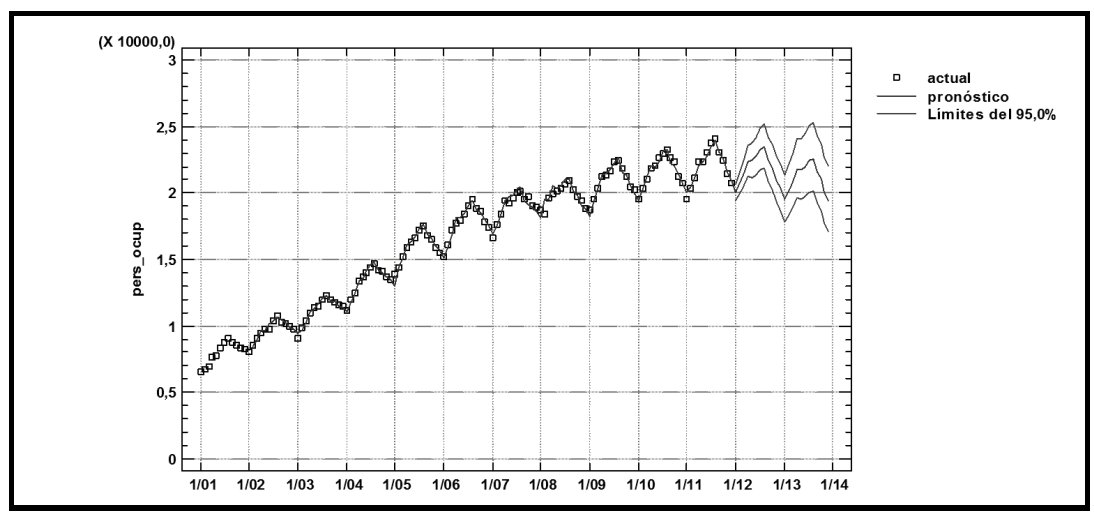

Fuente: Elaboración propia.

En consecuencia, esta previsión de pérdida de empleo manifiesta que el sector turístico no es inmune a la crisis económica. No obstante, y si se compara con la que presenta el propio sector agrario puede resultar, en cierto modo, no preocupante siempre y cuando se consiga hacer frente a esta situación.

Pero, cabe preguntarse cómo analizar la veracidad de estas predicciones. Una forma posible es a través de su comparación con los datos reales. En este sentido indicar que se ha estimado el modelo considerando parte de la información sobre el empleo (hasta el año 2011) y se ha utilizado la 
información de periodos posteriores (enero a noviembre de 2012) para contrastar la predicción efectuada.

De esta forma, se pretende verificar si la predicción se ajusta a la situación real y, para ello, se define un indicador que cuantifica la desviación o el error respecto a la información que realmente se ha producido en el periodo. En concreto, para el año 2012 se observa que, en los diferentes periodos mensuales, los valores estimados se aproximan a los valores reales, obteniendo una desviación relativamente reducida (inferior al 4\%) y, en todos los casos, los valores estimados se hallan dentro del intervalo de confianza establecido (95\%) (Tabla 5).

\section{Tabla 5}

Personal ocupado en turismo rural (Número, estimaciones al 95\%)

\begin{tabular}{|c|c|c|c|c|c|}
\hline Periodo & Estimación & Inferior & Superior & Real & $\begin{array}{c}\text { Desv/Real } \\
(\%)\end{array}$ \\
\hline $1 / 2012$ & 20.009 & 19.392 & 20.644 & 19.687 & $-1,64$ \\
\hline $2 / 2012$ & 20.705 & 19.926 & 21.514 & 19.928 & $-3,90$ \\
\hline $3 / 2012$ & 21.473 & 20.514 & 22.476 & 21.009 & $-2,21$ \\
\hline $4 / 2012$ & 22.397 & 21.271 & 23.583 & 22.050 & $-1,57$ \\
\hline $5 / 2012$ & 22.417 & 21.175 & 23.732 & 22.292 & $-0,56$ \\
\hline $6 / 2012$ & 22.758 & 21.391 & 24.212 & 22.638 & $-0,53$ \\
\hline $7 / 2012$ & 23.259 & 21.763 & 24.858 & 22.799 & $-2,02$ \\
\hline $8 / 2012$ & 23.430 & 21.830 & 25.148 & 23.366 & $-0,27$ \\
\hline $9 / 2012$ & 22.444 & 20.827 & 24.187 & 22.720 & 1,21 \\
\hline $10 / 2012$ & 21.878 & 20.224 & 23.667 & 22.137 & 1,16 \\
\hline $11 / 2012$ & 20.940 & 19.286 & 22.735 & 20.865 & $-0,35$ \\
\hline $12 / 2012$ & 20.331 & 18.661 & 22.151 & nd & nd \\
\hline
\end{tabular}

Fuente: Elaboración propia.

Asimismo, se realiza la predicción mensual del empleo en turismo rural para el periodo 2013 donde se observa, en todos los meses, un descenso en el personal ocupado respecto a los correspondientes meses del periodo 2012, situación que refleja una pérdida de empleo más acusada para este periodo (Tabla 6).

Tabla 6

Personal ocupado en turismo rural (Número, estimaciones al 95\%)

\begin{tabular}{|c|c|c|c|}
\hline Periodo & Estimación & Inferior & Superior \\
\hline $1 / 2013$ & 19.505 & 17.825 & 21.343 \\
\hline $2 / 2013$ & 20.158 & 18.352 & 22.142 \\
\hline $3 / 2013$ & 20.866 & 18.925 & 23.006 \\
\hline $4 / 2013$ & 21.726 & 19.634 & 24.041 \\
\hline
\end{tabular}


Tabla 6 (continuación)

Personal ocupado en turismo rural

(Número, estimaciones al 95\%)

\begin{tabular}{|c|c|c|c|}
\hline Periodo & Estimación & Inferior & Superior \\
\hline $5 / 2013$ & 21.707 & 19.549 & 24.104 \\
\hline $6 / 2013$ & 21.998 & 19.744 & 24.510 \\
\hline $7 / 2013$ & 22.443 & 20.077 & 25.087 \\
\hline $8 / 2013$ & 22.568 & 20.125 & 25.307 \\
\hline $9 / 2013$ & 21.580 & 19.185 & 24.274 \\
\hline $10 / 2013$ & 20.998 & 18.611 & 23.691 \\
\hline $11 / 2013$ & 20.062 & 17.730 & 22.701 \\
\hline $12 / 2013$ & 19.445 & 17.135 & 22.066 \\
\hline
\end{tabular}

Fuente: Elaboración propia.

\section{CONCLUSIONES}

El turismo rural es considerado, por muchos, como una iniciativa con cierta proyección futura en el área rural. La posibilidad de crear empleo e incrementar las rentas en muchas zonas puede reducir o evitar la despoblación en el ámbito rural, así como potenciar el interés por la conservación y protección del medio ambiente.

El turismo es una de las actividades económicas que mejor se está comportando en este periodo de crisis. De esta situación se ha beneficiado, en particular, el turismo rural que ha generado empleo en momentos en los que el entorno rural los pierde, aunque eso sí, con el apoyo de Organismos Públicos que han potenciado y mejorado el atractivo de la oferta turística.

Esta circunstancia ha llevado a amortiguar, en parte, la pérdida de empleo que viene padeciendo el campo español. Pero esta tendencia decreciente se mantiene y preocupa a las Administraciones Públicas que ven en ella peligrar la permanencia de la población rural en el campo. Por ello, son conscientes de que hay que buscar actividades, como el turismo, que posibiliten nuevos ingresos en el entorno rural.

En este contexto hay que tener en cuenta su comportamiento, con dos componentes que caracterizan el empleo en turismo rural, su tendencia (creciente y más o menos contenida) y su estacionalidad (con máximos en periodos estivales y mínimos en periodos invernales), pues ello afecta a la uniformidad en la renta que genera esta actividad.

Por lo tanto, la modelización del personal ocupado en turismo rural lleva a estimar un modelo ARIMA $(1,1,0) \mathrm{x}(0,1,1)_{12}$ con constante, con una previsión futura, grosso modo, de pérdida de empleo tanto para el año 2012 como para el periodo 2013. 
En este sentido se expresan ciertas informaciones ${ }^{2}$, que tachan de mala la campaña de este último verano del 2012, con caídas de ocupación de hasta el $20 \%$ respecto del 2011. Estas caídas se justifican por la fuerte bajada del cliente nacional aunque atenuadas por el crecimiento del número de extranjeros.

$\mathrm{Y}$ es que estas previsiones no son nada favorables, pues a la pérdida de renta de la población en los últimos tiempos (clientes potenciales) se une el incremento de precios que ha provocado la subida del IVA en estas actividades turísticas. Esta situación puede repercutir de una forma negativa en el sector y acentuar la pérdida de empleo en los próximos meses. Aún así, el turismo rural se está comportando mejor que otros sectores económicos.

\section{REFERENCIAS BIBLIOGRÁFICAS}

BIGOVIC, M. (2012). "Forecasting within Montenegrin tourism using Box-Jenkins methodology for seasonal Arima models. Tourism and Hospitality Management. Vol. 18 (1), pp. 1-18.

BRU, S. y USACH, J. (1987). "Análisis de series temporales de turismo de la Comunidad Valenciana". Revista Estadística Española, No 114, pp. 111-132.

BRUNET, I. y ALARCÓN, A. (2008). "El turismo rural en Cataluña. Estrategias empresariales". Revista Internacional de Sociología, Vol. LXVI, No 47 , pp. 141-165.

CALATRAVA, J. y SAYADI, S. (2004). "Permanencia de la actividad agraria y políticas de desarrollo rural: un análisis a partir de un seguimiento (19812001) a explotaciones agrarias en zonas de montaña del sureste español". Revista de Estudios Agrosociales y Pesqueros, № 204, pp. 207-218.

CAMILLERI, A. (1967). "La influencia de la movilidad de la mano de obra agrícola en la empresa agraria. Revista de Estudios Agrosociales № 58 , pp. 99135.

CÁNOVES, G.; HERRERA, L. y BLANCO, A. (2005). "Turismo rural en España: un análisis de la evolución en el contexto europeo". Cuadernos de Geografía, №. 77, pp. 41- 58.

CÁNOVES, G. y VILLARINO, M. (2000). "Turismo en espacio rural en España: actrices e imaginario colectivo". Anal. Geogr. 37, pp. 51-77.

CARAZO, L. 1982: "El turismo rural como recurso de la población agraria". Revista de Estudios Agrosociales, №. 120, pp. 118-130.

\footnotetext{
${ }^{2}$ Véase http://www.agroinformacion.com/noticias/65/turismo/51721/ el+turismo+rural+acaba+ un+verano + duro + con + caidas + de + ocupacion + de + hasta $+e l+20 . a s p x$
} 
CERCÓS, A. (1973): "El empleo en el sector agrario". Dirección General de Empleo del Ministerio de Trabajo. Comunicación pp. 7-21.

CHANG-JIU, L.; HSUEH-FANG, C. y TIAN-SHYUG, L. (2011): "Forecasting Tourism Demand using Time Series, Artificial Neural Networks and Multivariate Adaptive Regression Splines: Evidence from Taíwan. International Journal of Business Administration, Vol 2 (2), pp. 14-24.

CUÑADO, J.; ALBERIKO, L. y PÉREZ, F. (2011): "Modelling International Monthly Tourist in Spain". Revista de Estudios de Economía Aplicada. Vol. 29-3, pp. 723-736.

ESCUDERO, G. (1986): "Actividad, ocupación y productividad agraria en España: un análisis de la población y del empleo". Revista de Estudios Agrosociales Vol. 137, pp. 379-417.

FERNÁNDEZ-CAVADA, J.L.; MARTíN-FERNÁNDEZ, S. y ORTUÑO, S. (2007): "Evolución y perspectivas del mercado de trabajo agrario en España (19762004)". Revista Agrociencia No 41, pp. 241-251.

GARRIDO, L. y GONZÁLEZ, J. (1992): "El mercado de trabajo agrario: algunas cuestiones metodológicas y discusión de resultados". Revista de Estudios Regionales $N^{\circ} 31$, pp. 45-53.

GIL-ALANA, L.A. (2010): "Tourism in Africa. Time series persistence and the nature of shocks. Are they transitory or permanent? ". Working - Paper 6/10, Navarra Center for International Development, $27 \mathrm{p}$.

GIL-ALANA, L.A.; CUÑADO, J. y PÉREZ DE GRACIA, F. (2008): "Tourism in the Canary Islands: Forecasting using several time series models", Journal of Forecasting 27, pp. 621-636.

$\mathrm{GOH}, \mathrm{C}$. y LAW, R. (2002): "Modeling and forecasting tourism demand for arrivals with non-stationary seasonality and intervention", Tourism Management 23, pp. 499-414.

HOFF, J.C. (1983):"A practical guide to Box-Jenkins forecasting". Lifetime Learning Publications, $316 \mathrm{p}$.

HULTKRANTZ, L. y OLSSON, C. (1997): "Chernobyl Effects on Domestic and Inbound Tourism in Sweden - A Time Series Analysis. Environmental and Resource Economics 9, pp. 239-258.

JUANEDA, C. y RIERA, A. (2011). "La oportunidad de la investigación en economía del turismo". Revista de Estudios de Economía Aplicada. Vol. 29-3, pp. 711-722.

KADER, A. y CHIKR-EL-MEZOUAR, Z. (2011). "Time Series Modeling of Tourism in Southwest Algeria Case Study Bechar as Tourist Destination". International Research Journal of Finance and Economics, Issue 69, pp. 5156.

LILLO-BAÑULS, A. y CASADO-DÍAZ, J.M. (2011). "Capital humano y turismo: Rendimiento educativo, desajuste y satisfacción laboral". Revista de Estudios de Economía Aplicada. Vol. 29-3, pp. 755-780. 
LIM, C. y MCALEER, M. (2001). "Time series forecasts of international tourism demand for Australia". The Institute of Social and Economic Research Osaka University. Discussion Paper $n^{\circ}$ 533, 24 p.

MINISTERIO DE INDUSTRIA, ENERGÍA Y TURISMO (2008). "Plan Renove de Turismo". B.O.E. 1 diciembre 2008.

MINISTERIO DE MEDIO AMBIENTE Y MEDIO RURAL Y MARINO (2009): "Tendencias del turismo rural en España". Análisis y Prospectiva. Serie Agrlnfo. http://www.magrama.gob.es/es/ministerio/servicios-generales/serviciosde- informacion-y-participacion/agrinfo14_tcm7-161565.pdf

MINISTERIO DE MEDIO AMBIENTE Y MEDIO RURAL Y MARINO (2010): "Programa de desarrollo rural sostenible (2010-2014). http://www.magrama.gob.es/es/desarrollo-rural/temas/ley-para-el-desarrollosostenible-del-medio-rural/10._memoria_ambiental_conjunta_tcm79682.pdf.

MINISTERIO DE INDUSTRIA, ENERGÍA Y TURISMO (2012): "Plan Nacional e Integral de Turismo 2012-2015. http://www.minetur.gob.es/turismo/esES/ Documents/Plan\%20Nacional\%20e\%20Integral\%20de\%20Turismo\%202012_2015_v 4\%206.pdf

MONDEJAR, J.; VARGAS M.; MONDÉJAR, J.A. y LORENZO, C. (2007). "Extracción de señal y predicción en series turísticas". Cuadernos de Turismo. No 20. (2007), pp. 153-170.

MORLEY, C.L. (1993):"Forecasting Tourism Demand Using Extrapolative Time Series Methods. The Journal of Tourism Studies Vol. 4 (1), pp. 19-25.

PULIDO, J.I. y CÁRDENAS, P.J. (2011). "El turismo rural en España. Orientaciones estratégicas para una tipología aún en desarrollo". Boletín de la Asociación de Geógrafos Españoles 56, pp. 155-176

OCDE (1994). "Tourism strategies and Rural development". Paris OCDE/GD (94) 49, $94 \mathrm{p}$.

SONG, H.; WITT S.F. y JENSEN, T.C. (2003). "Tourism forecasting: accuracy of alternative econometric models". International Journal of Forecasting 19, pp. 123-141.

SONG, H., y Li, G. (2008). "Tourism demand modelling and forecasting-A review of recent research". Tourism Management 29, pp. 203-220.

VANDAELE, W. (1983). "Applied time series and Box-Jenkins models". Academic Press, $417 \mathrm{p}$.

WANHILL, S. (2011). "What Tourism Economists Do. Their Contribution to Understanding Tourism". Estudios de Economía Aplicada. Vol. 29 (3), pp. 679692.

YAGÜE, R.M. (2002). " Rural tourism in Spain". Annals of Tourism Research. Vol 29 (4), pp. 1101-1110. 
